\title{
Penetration Properties and Safety Aspects of Topically Applied Products
}

The leaves are falling ... and we present you the new edition of Skin Pharmacology and Physiology. Turbulences in the political and economic field are shaking the world, but we feel that our working environment is still relatively protected. However, changing economic coordinates might also touch our labs (e.g. funding sources, research programs) and urge us to make yet unpredicted strategic decisions. Isolation is not the right answer to external pressure! We would like to encourage you to seek more than ever for collaborations and active involvement in our society in order to create a framework where we can keep a small 'protected' area.

In this issue, Fortenbach et al. (Skin Pharmacol Physiol 2008;21:294-299) present a review paper on the correlation of orally and intravenously administered antibiotics. They provide insight into the role of physical and chemical properties in drug release into the skin compartment mainly based on a cantharidin blister model. Both orally and intravenously administered antibiotics display a linear correlation in the total concentration in the serum and interstitial fluid, indicating that serum concentration may be an important factor in dictating interstitial fluid concentration.

Safety of topical methimazole for the treatment of melasma was demonstrated by Kasraee and the Geneva group of Prof. Saurat (Skin Pharmacol Physiol 2008;21:300-305). They were able to show that long-term topical methimazole applications in melasma patients did not induce any significant changes in serum TSH, free thyroxine and free triiodothyronine levels. Topical methimazole was well tolerated and did not induce any significant cutaneous side effects. Thus, the authors suggest methimazole as a safe skin-depigmenting compound for topical treatment of skin hyperpigmentary disorders in humans.

A subject rarely studied was the irritative effect of bath oils in a comparative trial. Reuter et al. (Skin Pharmacol Physiol 2008;21:306-311) could show that not all bath oils have the same effect on epidermal barrier function and skin irritation. Their model was able to differentiate bath oil formulations. Evidence was provided for good skin tolerance of bath oils containing St. John's wort extract.

Lademann and Blume-Peytavi and their group (Skin Pharmacol Physiol 2008;21:312-317) were able to demonstrate the changes in hair cross sections during glucocorticosteroid treatment. Significant differences in the hair cross section could be detected during treatment, while the shape of the hairs was not influenced. The authors suggest optical coherence tomography as a suitable, noninvasive and low-cost measuring technique that can be applied for doping control and screening.

Monti et al. (Skin Pharmacol Physiol 2008;21:318-325) compared skin permeation and distribution profiles of two sunscreens using reconstructed human skin and hairless rat skin. Different distribution profiles of the tested formulations could be ascribed to the different lipid compositions of reconstructed human skin and rat skin. Since the physicochemical characteristics of reconstructed skin are closer to those of human skin, the authors suggest that reconstructed human skin models could be suitable to replace human skin in 'in vitro testing'.

Otto et al. (Skin Pharmacol Physiol 2008;21:326-334) showed that neither $10 \%$ dimethyl isosorbide nor $10 \%$ diethylene glycol monomethyl ether significantly enhanced the skin permeation of the various lipophilic active ingredients or the uptake into the skin. They hypothesized that the effect of dimethyl isosorbide and diethylene glycol monomethyl ether on the solubility of the active ingredients in the skin was counteracted by a simultaneous reduction of the thermodynamic activity in the formulation.

Now we would like to invite you to join our society (put the budget in for 2009!) and get involved in preparing the summer meeting in Washington, D.C.

Joachim Fluhr, President of the ISP Jürgen Lademann, Editor

\begin{tabular}{ll}
\hline KARGER & @ 2008 S. Karger AG, Basel \\
Fax $+41660-5527 / 08 / 0216-0293 \$ 24.50 / 0$ \\
$\begin{array}{l}\text { E-Mail karger@karger.ch } \\
\text { www.karger.com }\end{array}$ & $\begin{array}{l}\text { Accessible online at: } \\
\text { www.karger.com/spp }\end{array}$
\end{tabular}

